PROCEEDINGS OF THE

AMERICAN MATHEMATICAL SOCIETY

Volume 128, Number 9, Pages 2683-2685

S 0002-9939(00)05348-X

Article electronically published on February 25, 2000

\title{
GEOMETRIC PROPERTIES OF THE SOLUTIONS OF A HELE-SHAW TYPE EQUATION
}

\author{
KONSTANTIN KORNEV AND ALEXANDER VASIL'EV
}

(Communicated by Albert Baernstein II)

\begin{abstract}
This article deals with the application of the methods of geometric function theory to the investigation of the free boundary problem for the equation describing flows in an unbounded simply-connected plane domain. We prove the invariance of some geometric properties of a moving boundary.
\end{abstract}

The global behavior of solutions in dynamical models of mathematical physics with free boundaries is complicated, foremost due to the irregular behavior of the boundary. The properties of this free boundary which remain the same are of constant interest. In this paper we study a plane dynamical problem describing the two-dimensional flow of fluid in an unbounded simply-connected plane domain. The classical case of similar dynamics and different conditions is represented by the Hele-Shaw equation (see [1, 3]-[5]). In our paper the methods of geometric function theory are applied to the investigation of hereditary geometric properties of the free boundary which depend on those of the initial one (see also [2]). Physically, this mathematical model describes the process when an infinite crystal with certain plane geometric properties is laved by the flow of an incompressible fluid. This problem relates to the Hele-Shaw model in case of injection with an obstacle. It can be reduced [2], 3 to a non-linear boundary one for analytic functions. Let $z=f(\zeta, t)$ for any fixed $t \geq 0$ be an analytic univalent mapping of the upper halfplane $H:=\{\zeta: \Im m \zeta>0\}$ to an unbounded domain of an incompressible fluid flow in the physical plane $(z)$ that being extended onto the real axis satisfies the Hele-Shaw type equation

$$
\Im m \frac{\partial f}{\partial t} \cdot \overline{\left(\frac{\partial f}{\partial \zeta}\right)}=\beta<0, \quad \Im m \zeta=0,
$$

with the series expansion in the neighbourhood of infinity $f(\zeta, \cdot)=\zeta^{2}+a_{1} \zeta+a_{0}+$ $a_{-1} / \zeta+\ldots$. The moving boundary of the flow domain in the mathematical model considered, $D(t)$, can be parametrized by the curve $f(\partial H, t)$. In (1) we assume that the power of the flow $\beta$ is constant. Otherwise, it can be reduced to the constant

Received by the editors May 26, 1998 and, in revised form, October 27, 1998.

1991 Mathematics Subject Classification. Primary 35Q35; Secondary 30C45.

Key words and phrases. Free boundary, Hele-Shaw equation, convex function in the positive direction.

The authors were supported in part by the Russian Foundation for Basic Research, Grants \#98-01-00842, \#98-15-96002.

(C)2000 American Mathematical Society 
one by change of variables. We pose the initial condition

$$
f(\zeta, 0)=f_{0}(\zeta), \quad \zeta \in H,
$$

generated by an initial flow domain with boundary $D(0)$. It is known [5] that solutions of (1) exist locally in time.

A reduction based on the Cauchy integral representation of (1) can be written as

$$
\pi \frac{\partial f}{\partial t}=\frac{\partial f}{\partial \zeta} \int_{-\infty}^{\infty} \frac{\beta d x}{\left|f^{\prime}(x, t)\right|^{2}(x-\zeta)}, \quad \zeta=x+i y, y>0 .
$$

Here $f^{\prime}(x, t)=\partial f / \partial \zeta$ at the point $x$.

We now define the geometric properties of $D(0)$ natural for the problem in question. A simply-connected domain $D$ of hyperbolic type on the extended complex plane $\overline{\mathbb{C}}$ is said to be convex in the positive direction of the real axis $\mathbb{R}^{+}$if its complement can be covered by the family of non-intersecting rays that are parallel and codirected with $\mathbb{R}^{+}$. A holomorphic univalent mapping $f(\zeta), \zeta \in H$, is said to be convex in the positive direction if $f(H)$ is as above. The criterion of this property is provided by the inequality

$$
\Im m f^{\prime}(\zeta)>0, \quad \zeta \in H .
$$

It is easy to construct an example of the normalized function above $f(\zeta)=\zeta^{2}+$ $2 i \zeta+1$. If we consider $f$ in the closure of $H$, then the equality sign in (4) can be reached only in case $y=0$. The level lines, i.e., the $f$-images of the lines $y=$ const, $y>0$, also bound domains convex in the positive direction if the function $f$ is convex in the positive direction.

Theorem. Let $D(0)$ (and so that for $f(\zeta, 0)$ ) be a domain convex in the positive direction. Then for all $t \geq 0$ such that there exist solutions of the equation (1) the family of functions $f(\zeta, t)$ and the family of domains $D(t)$ preserve the same property of convexity.

Proof. We assume the contrary. Suppose that there exist $t_{0} \geq 0$ and $\zeta_{0} \in \mathbb{R}$ such that

$$
\Im m f^{\prime}\left(\zeta_{0}, t_{0}\right)=0,
$$

where $f^{\prime}(\zeta, t)=\partial f(\zeta, t) / \partial \zeta$. This means that the mapping $f\left(\zeta, t_{0}\right)$ is critical for the property of convexity in the positive direction. Let $t_{0}$ be the minimal such quantity. Without loss of generality we suppose that $f^{\prime}\left(\zeta_{0}, t_{0}\right)>0$.

First we show that $f^{\prime \prime}\left(\zeta_{0}, t_{0}\right) \neq 0$. If not, then the point $\zeta_{0}$ is the branch point of the function $f^{\prime}\left(\zeta, t_{0}\right)$ and in a neighbourhood of this point in $H$ the quantity $\arg f^{\prime}\left(\zeta, t_{0}\right)$ admits either positive or negative values. This contradicts the assumption that the function $f\left(\zeta, t_{0}\right)$ is convex in the positive direction.

Since $\zeta_{0}$ is the critical point in which the equality sign in (4) is realized, the image of the upper half-plane $H$ under the mapping $f^{\prime}\left(\zeta, t_{0}\right)$ touches the real axis at the point $f^{\prime}\left(\zeta_{0}, t_{0}\right)$. Thus, the following statements are true:

$$
\left.\frac{\partial}{\partial x} \arg f^{\prime}\left(x, t_{0}\right)\right|_{x=x_{0}}=0,\left.\quad \frac{\partial}{\partial y} \arg f^{\prime}\left(x_{0}+i y, t_{0}\right)\right|_{y=0} \geq 0,
$$


where $\zeta=x+i y, \zeta_{0}=x_{0}, y \geq 0$. Calculation of the right-hand sides of these formulas leads to the following:

$$
\Im m \frac{f^{\prime \prime}\left(x_{0}, t_{0}\right)}{f^{\prime}\left(x_{0}, t_{0}\right)}=0, \quad \Re e \frac{f^{\prime \prime}\left(x_{0}, t_{0}\right)}{f^{\prime}\left(x_{0}, t_{0}\right)} \geq 0 .
$$

Denote

$$
p(\zeta, t):=\frac{\beta}{\pi} \int_{-\infty}^{\infty} \frac{d x}{\left|f^{\prime}(x, t)\right|^{2}(x-\zeta)} .
$$

From equation (3) we obtain

$$
\frac{\partial}{\partial t} \arg f^{\prime}(\zeta, t)=\Im m\left(\frac{f^{\prime \prime}(\zeta, t)}{f^{\prime}(\zeta, t)} p(\zeta, t)+\frac{\partial p(\zeta, t)}{\partial \zeta}\right) .
$$

Integrating by parts we deduce that

$$
\frac{\partial p(\zeta, t)}{\partial \zeta}=\frac{\beta}{\pi} \int_{-\infty}^{\infty} \frac{d x}{\left|f^{\prime}(x, t)\right|^{2}(x-\zeta)^{2}}=-\frac{2 \beta}{\pi} \int_{-\infty}^{\infty} \frac{\Re e \frac{f^{\prime \prime}(x, t)}{f^{\prime}(x, t)} d x}{\left|f^{\prime}(x, t)\right|^{2}(x-\zeta)}
$$

Using the Cauchy representation and equality (5) we obtain finally that

$$
\left.\frac{\partial}{\partial t} \arg f^{\prime}\left(\zeta_{0}, t\right)\right|_{t=t_{0}}=-\frac{\beta}{\left|f^{\prime}\left(\zeta_{0}, t_{0}\right)\right|^{2}} \Re e \frac{f^{\prime \prime}\left(\zeta_{0}, t_{0}\right)}{f^{\prime}\left(\zeta_{0}, t_{0}\right)} .
$$

Since $f^{\prime \prime}\left(\zeta_{0}, t_{0}\right) \neq 0$ and (6), the right-hand side of (7) is strictly positive. Therefore, $\left.\frac{\partial}{\partial t} \arg f^{\prime}\left(\zeta_{0}, t\right)\right|_{t=t_{0}}$ is also strictly positive in some neighbourhood of $\zeta_{0}$ and near other points possessing such critical properties. Thus, for $t>t_{0}$ (close to $\left.t_{0}\right)$ in this neighbourhood the following inequality is true: $\Im m f^{\prime}(\zeta, t)>0$. For all $\zeta$ outside the neighbourhood mentioned the same inequality is valid because $\Im m f^{\prime}\left(\zeta, t_{0}\right) \geq$ $\delta>0$ for such $\zeta$. This contradicts the assumption and ends the proof.

\section{REFERENCES}

[1] C.M.Elliot, J.R.Ockendon, Weak and variational methods for moving boundary problem, Pitman, London, 1992. MR 83i:35157

[2] Yu.E.Hohlov, D.V.Prokhorov, A.Yu.Vasil'ev, On geometric properties of free boundaries in the Hele-Shaw flows moving boundary problem, Lobachevskii J. Math. 1 (1998), 3-13.

[3] S.D.Howison, Complex variable methods in Hele-Shaw moving boundary problems, Europ. J. Appl. Math. 3 (3) (1992), 209-224. MR 94f:76025

[4] S.D.Howison, Yu.E.Hohlov, On the classification of solutions to the zero-surface-tension model for Hele-Shaw free boundary flows, Quart. Appl. Math. 54 (4) (1994), 777-789. MR 94j:76070

[5] M.Reissig, L. von Wolfersdorf, A simplified proof for a moving boundary problem for HeleShaw flows in the plane, Ark. Mat. 31 (1) (1993), 101-116. MR 94m:35250]

Institute of Problems in Mechanics, Russian Academy of Sciences, Moscow, Russia E-mail address: kidin@ipm.msk.su

Departamento de Matemáticas, Universidad de los Andes, Bogotá, Colombia

E-mail address: avassill@uniandes.edu.co 